\title{
BRINQUEDO TERAPÊUTICO NO ALÍVIO DA DOR EM CRIANÇAS HOSPITALIZADAS
}

\section{Ana Carolina de Almeida Gomes ${ }^{1}$, Aline Teixeira Marques Figueiredo Silva ${ }^{2}$, Carolina Magalhães dos Santos $^{3}$ \& Thaís Aparecida de Castro Palermo ${ }^{3}$}

\begin{abstract}
RESUMO
GOMES, A.C.A.; SILVA, A.T.M.F.; SANTOS, C.M. \& PALERMO, T.A.C. Brinquedo terapêutico no alívio da dor em crianças hospitalizadas Perspectivas Online: Biológicas \& Saúde. v. 9, n 29, p.33-42, 2019.
\end{abstract}

A hospitalização de uma criança pode ser uma das maiores fontes de ansiedade tanto para a própria criança, quanto para os familiares e profissionais de saúde. $\mathrm{O}$ Brinquedo Terapêutico Instrucional (BTI) tem como finalidade preparar a criança para a hospitalização, dramatizando a situação a ser vivenciada. A pesquisa tem como objetivo avaliar a eficácia do uso do BTI para o alívio de dor em crianças hospitalizadas, através da análise das manifestações demonstradas pela criança nos procedimentos dolorosos e como objetivos específicos, avaliar a dor apresentada pelas crianças durante a realização de procedimentos dolorosos, antes e após o uso do BTI e discutir o uso do BTI pela enfermagem, em crianças hospitalizadas. Trata-se de uma pesquisa descritiva, exploratória, de abordagem quantitativa. A pesquisa foi realizada em um hospital público de grande porte do município de Campos dos Goytacazes, Rio de Janeiro. A amostra foi selecionada por conveniência e foram incluídas todas as crianças internadas no setor de pediatria e excluídas aquelas que estiverem fora da faixa etária de 2 a 10 anos. Os dados foram coletados por meio da observação da criança durante a realização dos procedimentos dolorosos (curativos e punções venosas) e foram avaliados de forma descritiva. Evidenciou-se uma redução da dor referida pelas crianças, obtendo uma redução da dor em $96,9 \%$ dos procedimentos realizados. Conclui-se que o BTI contribui na minimização do estresse decorrente do processo de hospitalização e proporciona um melhor relacionamento entre a equipe de saúde e a criança.

Palavras-chave: Jogos e brinquedos; Criança hospitalizada; Enfermagem Pediátrica. 


\begin{abstract}
Hospitalization of a child can be a major source of anxiety for both the child and family members and health care professionals. The Instructional Therapeutic Toy (BTI) aims to prepare the child for hospitalization, dramatizing the situation to be experienced. The objective of this research was to evaluate the effectiveness of BTI for the relief of pain in hospitalized children by analyzing the manifestations demonstrated by the child in the painful procedures and as specific objectives to evaluate the pain presented by the children during painful procedures, before and after the use of BTI and discuss the use of BTI by nursing in hospitalized children. This is a descriptive, exploratory, quantitative approach. The research was conducted in a large public

hospital in the municipality of Campos dos Goytacazes, Rio de Janeiro. The sample was selected for convenience and included all the children hospitalized in the pediatric department and excluded those who are outside the age range of 2 to 10 years. The data were collected through the observation of the child during the accomplishment of the painful procedures (dressings and venous punctures) and were evaluated in a descriptive way. A reduction of the pain reported by the children was evidenced, obtaining a reduction of pain in $96.9 \%$ of the procedures performed. It is concluded that BTI contributes to the minimization of stress resulting from the hospitalization process and provides a better relationship between the health team and the child.
\end{abstract}

Keywords: Play and playthings; Child, hospitalized; Pediatric nursing.

\footnotetext{
${ }^{1}$ Institutos Superiores de Ensino do CENSA -ISECENSA - Aluna do curso de Enfermagem - Rua Salvador Correa, 139, Centro, Campos dos Goytacazes, RJ, CEP: 28035-310, Brasil.

2 Pesquisadora do Laboratório de Estudos Hospitalares e Terapia Intensiva - LAEHTI/ ISECENSA

${ }^{3}$ Pesquisadora do Laboratório de Estudos em Saúde Pública - LAESP/ISECENSA

(*)e-mail: thaisacpalermo@gmail.com

Data de recebimento: 20/03/2019 Aceito para publicação: 30/04/2019
} 


\section{INTRODUÇÃO}

O brincar é um fator essencial para o desenvolvimento da criança e está presente em todas as fases da vida dela. Tem como finalidade desenvolver a expressão da criança, pois por meio das brincadeiras ocorre a manifestação de seus anseios, conflitos, desejos e suas experiências vividas (FONTES et al., 2010; SOUSA et al., 2015).

A inserção das atividades lúdicas no cuidado em Enfermagem Pediátrica, como o Brinquedo Terapêutico Instrucional, pode contribuir na diminuição da dor, tensão, raiva, estresse, frustração, conflito e ansiedade decorrentes da hospitalização. O brinquedo comum torna-se terapêutico a partir do momento que promove o bem-estar psicofisiológico da criança (MAIA et al., 2011).

Pode ser classificado de três formas: o brinquedo dramático, o brinquedo instrucional e o brinquedo capacitador de funções fisiológicas. O Brinquedo Terapêutico Instrucional (BTI) tem como finalidade preparar a criança para a hospitalização, dramatizando a situação a ser vivenciada (ROCHA et al., 2012).

O preparo da criança que será submetida aos procedimentos hospitalares através do Brinquedo Terapêutico Instrucional (BTI) é uma ferramenta utilizada na tentativa de amenizar o sofrimento e os traumas causados pelos procedimentos invasivos, além de ser uma intervenção eficaz que permite à criança saber o que deve esperar e como deve participar durante o procedimento; compreender a finalidade e a necessidade do procedimento; manipular material previamente; envolver-se na situação ao invés de ser tratada como objeto passivo e posteriormente estabelecer relação de confiança com o adulto e o profissional (MEDEIROS et al., 2009).

A hospitalização de uma criança pode ser uma das maiores fontes de ansiedade tanto para a própria criança quanto para os familiares e profissionais de saúde (SOUZA et al., 2012). O paciente hospitalizado possui diversos fatores que podem ocasionar mudanças em seu estado psicológico e com isso alterar ou intensificar sua representação de dor devido ao medo de se submeter a certos procedimentos, medo da morte e de estar longe da família (FORTUNATO et al., 2013).

Uma assistência de enfermagem de qualidade à criança exige além da execução adequada da técnica e do domínio dos conhecimentos relacionados à determinada patologia, um atendimento holístico, onde a criança e sua família são assistidas como um todo, atendendo as suas necessidades emocionais, estabelecendo vínculos com ela e a família, compreendendo-a em todas as suas fases de desenvolvimento e especialmente quando vivencia um processo de doença, garantindo a promoção e a recuperação da sua saúde (ROCHA et al., 2012).

Contudo, a assistência a criança hospitalizada deve considerar não apenas o cuidado físico e/ou tratamento clínico. Existem recursos ou medidas que proporcionam uma melhor qualidade na assistência prestada, como explicar os motivos da hospitalização, preparar a criança para as intervenções que irão ser feitas e utilizar o BTI como parte da assistência prestada (KICHE; ALMEIDA, 2009).

Sendo assim, este estudo teve o objetivo de avaliar a eficácia do uso do brinquedo terapêutico instrucional para o alívio da dor em crianças hospitalizadas, através da análise das manifestações demonstradas pela criança nos procedimentos dolorosos. 


\section{METODOLOGIA}

Trata-se de um estudo descritivo, exploratório, de abordagem quantitativa, realizado no setor de pediatria de um hospital público de grande porte do município de Campos dos Goytacazes, RJ. Este hospital foi selecionado por possuir um espaço para o brincar terapêutico e pela demanda de crianças atendidas.

A amostra foi selecionada por conveniência, sendo incluídas todas as crianças internadas no setor de pediatria e excluídas aquelas que estiveram fora da faixa etária de 2 a 10 anos. Os dados foram coletados por meio da observação da criança durante a realização dos procedimentos dolorosos em dois momentos.

No primeiro momento, foram registrados os dados da criança na ficha de identificação (iniciais do nome do responsável pela criança, idade da criança, sexo, procedimento realizado, escore de dor antes e depois do BTI) e logo após foi observado o comportamento e as reações da criança referente à dor durante a realização dos procedimentos dolorosos (curativos e punções venosas). Neste momento, foi utilizada a escala de faces de dor Wong e Baker e registrado o valor apresentado pela criança antes do uso do BTI.

Após o término dos procedimentos foi realizada uma sessão de BTI, sendo demonstrado para a criança, em uma boneca, o respectivo procedimento realizado nela. A sessão de BTI foi realizada em um espaço de brincar no hospital denominado "brinquedoteca", porém devido à climatização do espaço ou crianças impossibilitadas de se locomover ao local, algumas sessões foram realizadas no leito do paciente.

Ao final da demonstração a criança foi convidada para repetir a brincadeira, possibilitando a identificação dos aspectos erroneamente compreendidos e esclarecendo-os, se necessário. Os materiais utilizados na sessão de BTI incluíram: uma boneca e os materiais referentes ao procedimento realizado na criança. Os materiais usados na sessão de BTI foram higienizados antes e após as sessões, com gaze e álcool a 70\%.

No dia seguinte, antes da realização dos procedimentos (curativos e punções venosas), foi repetida a sessão do BTI. Durante a execução do procedimento doloroso foi observado novamente os comportamentos e as reações da criança referente à dor utilizando a escala de faces de dor Wong e Baker, sendo registrado o valor apresentado pela criança na ficha de identificação da criança.

Os dados coletados foram digitados no Excel e posteriormente, analisados no software Statistical Package for the Social Sciences (SPSS), versão 24.0. Este estudo foi submetido ao Comitê de Ética em Pesquisa (CEP) dos Institutos Superiores do Censa, sendo aprovado em 08/12/2017, cujo número de protocolo é 80509417.1.0000.5524. Todos os responsáveis pelos participantes assinaram o Termo de Consentimento Livre e Esclarecido (TCLE), conforme a Resolução 466/12 do Conselho Nacional de Saúde (BRASIL, 2012).

\section{RESULTADOS}

Fizeram parte deste estudo 32 crianças internadas no setor de pediatria. A amostra foi predominantemente feminina $(53,1 \%)$ com idade média de 5 anos ( $\mathrm{DP}=2,1$ anos). Vide gráfico 1

Persp. online: biol. \& saúde, Campos dos Goytacazes, 29 (9),33- 422019

seer.perspectivasonline.com.br 
abaixo.

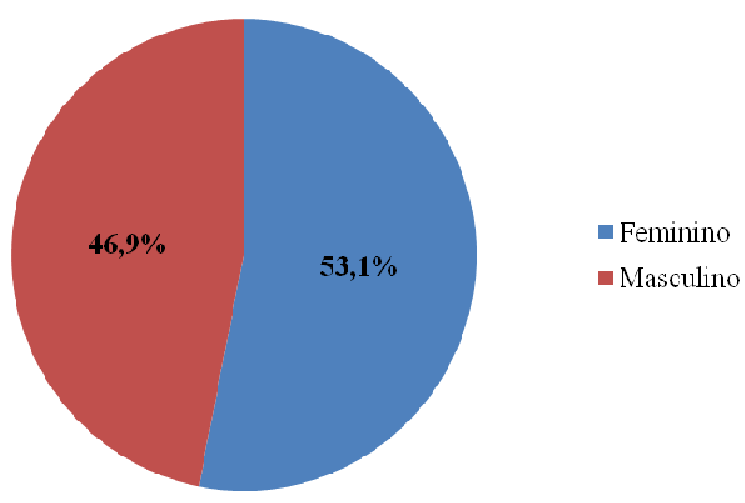

Figura 1: Caracterização da amostra por sexo, Campos dos Goytacazes/RJ 2018.

Para a aplicação do método investigado foram selecionados dois procedimentos de enfermagem: curativos (de queimaduras, escoriações e traumas realizados diariamente) e punções venosas. Desses, $71,9 \%$ eram punção venosa e $28,1 \%$ curativo, conforme o gráfico 2 a seguir.

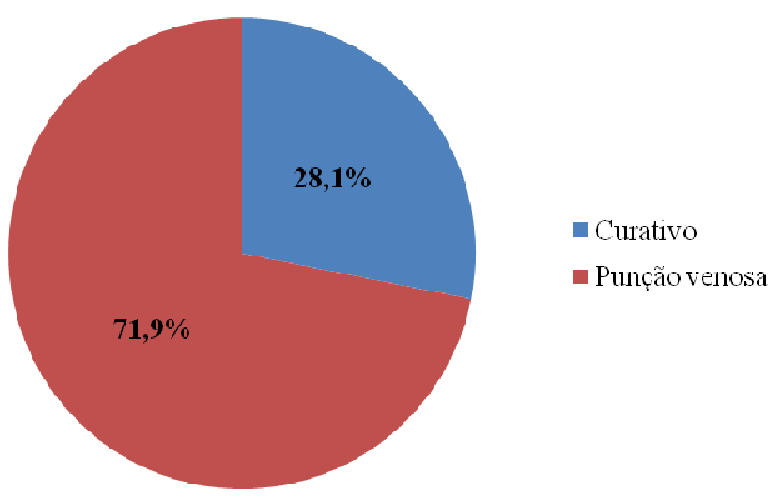

Figura 2 - Procedimentos realizados nas crianças, Campos dos Goytacazes/RJ, 2018.

Considerando a intensidade da dor sentida pela criança durante a realização dos procedimentos, antes da utilização do Brinquedo Terapêutico Instrucional (BTI), a maioria delas referiu escore $3(53,1 \%)$, evidenciando dor moderada. Enquanto após a sessão, predominaram os escores $1(37,5 \%)$ e $2(37,5 \%)$ evidenciando a dor muito leve e leve, respectivamente, de acordo com a tabela 1 a seguir. 
Tabela 1: Escores de dor referidos pelas crianças durante o procedimento realizado, antes e após a sessão de BTI, Campos dos Goytacazes/RJ, 2018

\begin{tabular}{lllll}
\hline Escore de dor & \multicolumn{2}{c}{ Antes do BTI } & \multicolumn{2}{c}{ Depois do BTI } \\
\cline { 2 - 6 } & n & \% & n & \% \\
\hline 0 & 0 & 0,0 & 3 & 9,4 \\
1 & 0 & 0,0 & 12 & 37,5 \\
2 & 5 & 15,6 & 12 & 37,5 \\
3 & 17 & 53,1 & 4 & 12,5 \\
4 & 7 & 21,9 & 1 & 3,1 \\
5 & 3 & 9,4 & 0 & 0,0 \\
Total & $\mathbf{3 2}$ & $\mathbf{1 0 0 , 0}$ & $\mathbf{3 2}$ & $\mathbf{1 0 0 , 0}$ \\
\hline
\end{tabular}

Os procedimentos foram avaliados isoladamente e foi observado que durante a realização do curativo, 55,6\% referiram escore 3, evidenciando dor moderada, antes da utilização do BTI. Após a realização da sessão de Brinquedo Terapêutico Instrucional (BTI), 33,3\% das crianças referiram escore 1 e 33,3\% referiram escore 2, evidenciando dor muito leve e leve, respectivamente. Vide tabela 2 abaixo.

Tabela 2- Escore de dor referido pelas crianças durante a realização do curativo, antes e após o uso do BTI, Campos dos Goytacazes/RJ, 2018

\begin{tabular}{llllll}
\hline Escore de dor & \multicolumn{2}{c}{ Antes do BTI } & \multicolumn{2}{c}{ Depois do BTI } \\
\cline { 2 - 6 } & n & \% & n & \% \\
\hline 0 & 00 & 0,0 & 00 & 0,0 \\
1 & 00 & 0,0 & 03 & 33,3 \\
2 & 00 & 0,0 & 03 & 33,3 \\
3 & 05 & 55,6 & 02 & 22,2 \\
4 & 02 & 22,2 & 01 & 11,1 \\
5 & 02 & 22,2 & 00 & 0,0 \\
Total & $\mathbf{0 9}$ & $\mathbf{1 0 0 , 0}$ & $\mathbf{0 9}$ & $\mathbf{1 0 0 , 0}$ \\
\hline
\end{tabular}

Durante a realização do procedimento de punção venosa, 52,2\% referiram escore 3, evidenciando dor moderada, antes da utilização do BTI. Após a realização da sessão de Brinquedo Terapêutico Instrucional (BTI), 39,1\% das crianças referiram escore 1 e 39,1\% referiram escore 2, evidenciando dor muito leve a leve, respectivamente, segundo a tabela 3 a seguir. 
Tabela 3- Escore de dor referido pelas crianças durante a realização da punção venosa, antes e após o uso do BTI, Campos dos Goytacazes/RJ, 2018

\begin{tabular}{llllll}
\hline Escore de dor & \multicolumn{2}{c}{ Antes do BTI } & \multicolumn{2}{c}{ Depois do BTI } \\
\cline { 2 - 6 } & $\mathbf{n}$ & $\mathbf{\%}$ & $\mathbf{n}$ & $\mathbf{\%}$ \\
\hline 0 & 00 & 0,0 & 03 & 13,0 \\
1 & 00 & 0,0 & 09 & 39,1 \\
2 & 05 & 21,7 & 09 & 39,1 \\
3 & 12 & 52,2 & 02 & 8,7 \\
4 & 05 & 21,7 & 00 & 0,0 \\
5 & 01 & 4,3 & 00 & 0,0 \\
Total & $\mathbf{2 3}$ & $\mathbf{1 0 0 , 0}$ & $\mathbf{2 3}$ & $\mathbf{1 0 0 , 0}$ \\
\hline
\end{tabular}

Comparando os escores de dor antes e após o uso do BTI referidos pelas crianças durante a realização dos procedimentos, 96,9\% referiram escores menores após a realização da sessão de Brinquedo Terapêutico Instrucional e apenas uma delas $(3,1 \%)$ continuou a referir o mesmo escore de dor da escala de faces conforme apresentado na tabela 4 abaixo. Todas essas diferenças observadas foram estatisticamente significativas pelo teste de Wilcoxon $(a=0,000)$, evidenciando uma redução da dor, após a utilização do método BTI.

Tabela 4: Comparação entre os escores de dor apresentados pela mesma criança antes e após a sessão do BTI, Campos dos Goytacazes/RJ, 2018

\begin{tabular}{lll}
\hline Comparação entre escore de dor antes e após o BTI* & \multicolumn{2}{l}{ Crianças } \\
& $\mathbf{n}$ & $\mathbf{\%}$ \\
\cline { 2 - 3 } & 31 & 96,9 \\
Escore de dor diminuiu após o BTI & 00 & 0,0 \\
Escore de dor aumentou após o BTI & 01 & 3,1 \\
Mesmo escore após o BTI & $\mathbf{3 2}$ & $\mathbf{1 0 0 , 0}$ \\
Total &
\end{tabular}

*Teste de Wilcoxon: $\mathrm{a}=0,000$

\section{DISCUSSÃO}

Neste estudo, evidenciou-se uma redução da dor referida pelas crianças durante a realização dos procedimentos (curativos e punções venosas), sendo relatado antes do uso do Brinquedo Terapêutico Instrucional (BTI), em sua maioria, um escore maior e após, as mesmas referiram escores menores, obtendo uma redução em $96,9 \%$ dos procedimentos realizados. Tal resultado evidencia a eficácia do uso do BTI na diminuição da dor durante os procedimentos realizados pela enfermagem nesses pacientes.

Um estudo realizado na unidade cirúrgica pediátrica com 34 crianças internadas para cirurgias de pequeno e médio porte apresentou resultados que corroboram com os aqui encontrados, sendo positivos para o uso do BTI durante o curativo pós-cirúrgico, havendo uma diminuição de 97\% no escore de dor apresentado pela criança após a sessão do brinquedo terapêutico (KICHE e

Persp. online: biol. \& saúde, Campos dos Goytacazes, 29 (9),33- 422019 seer.perspectivasonline.com.br 
ALMEIDA, 2009).

Destaca-se a importância da utilização do BTI ao possibilitar uma interação eficaz entre o adulto e a criança, promovendo maior aceitação e adaptação da criança ao processo de hospitalização, tornando-o mais agradável. O BTI promove o alívio da dor ao reduzir o medo e a ansiedade da criança, melhorando o seu estado de humor (FONTES et al., 2010).

No presente estudo, observou-se um resultado mais eficaz no que se refere à redução da dor após a utilização do BTI entre os indivíduos que realizaram o procedimento de punção venosa em relação aos indivíduos que realizaram o procedimento de curativo, ou seja, entre os indivíduos que realizaram o curativo o escore de dor após o uso do BTI foi maior em relação aos relatados pelos indivíduos que realizaram a punção venosa, demonstrando uma diminuição maior da dor nesses pacientes.

A hipótese é de que esses curativos de feridas de maior gravidade (como por exemplo, as queimaduras) resultem em maior nível de dor e por isso a redução do escore foi maior entre os indivíduos que foram submetidos à punção venosa que, segundo DANSKI et al. (2016) é um procedimento mais simples e cotidiano.

Segundo Pontes (2015), as crianças que são preparadas com o BTI se sentem mais tranquilas, passam a colaborar com o procedimento e obtém a oportunidade de lidar com a experiência dolorosa antes de vivenciá-la, aliviando também o estresse.

Através da brincadeira é dada a oportunidade para a criança exteriorizar seus sentimentos frente a situações novas e ameaçadoras. O brincar irá proporcionar o alívio das tensões, mostrando ser um instrumento essencial no cuidado pediátrico, conforme apresentado na revisão integrativa de literatura realizada com 22 artigos publicados entre 2005 e 2011 retirado da base de dados do Scielo e LILACS, utilizando o descritor Brinquedo terapêutico (CRUZ et al., 2013)

Estudo realizado com 8 acompanhantes de crianças a serem submetidas a punção venosa em um ambulatório pediátrico, revelou que os pais aprovam o uso do BTI e consideram seus efeitos satisfatórios e benéficos para a criança, pois de acordo com os mesmos, o seu uso tranquiliza e distrai a criança, diminuindo o seu medo, favorecendo a compreensão e a aceitação do procedimento e promovendo sua segurança (CONCEIÇÃO et al., 2011).

De acordo com SOUZA et al. (2012), o uso do BTI é uma ferramenta importante para entender melhor o paciente e diagnosticar suas necessidades, fornecendo recursos para $o$ profissional reconhecer as reais dificuldades e necessidades da criança e planejar a assistência de enfermagem adequada.

O Conselho Federal de Enfermagem (2004) afirma que o BTI pode ser amplamente utilizado por estes profissionais como uma atividade recomendada e regulamentada pelo órgão de classe, disponibilizando desta forma um instrumento com a eficácia cientificamente provada para a melhoria da assistência prestada pela enfermagem, garantindo uma redução do desconforto e maior segurança aos pacientes pediátricos. 


\section{CONSIDERAÇÕES FINAIS}

Neste estudo, o BTI se mostrou mais eficaz na redução da dor durante a realização da punção venosa quando comparado a redução da dor durante a realização do curativo. Apesar disso, ambos os procedimentos apresentaram redução da dor após a utilização do BTI, sendo este considerado uma estratégia importante para o cuidado de enfermagem pediátrica.

Foram realizadas apenas duas sessões de BTI por criança, todavia recomenda-se o uso contínuo do BTI por todos os profissionais de saúde e principalmente pela enfermagem para que os benefícios sejam constantes e duradouros.

Recomenda-se a realização de estudos que avaliem a eficácia do mesmo tanto na punção venosa como no curativo e em outros procedimentos que possam causar estresse e desconforto ao paciente pediátrico hospitalizado, contribuindo desta forma com a ampliação das possibilidades de seu uso nesse contexto.

O BTI possui vários benefícios tanto para a criança, quanto para os acompanhantes e os profissionais de saúde. Contribui na minimização do estresse decorrente do processo de hospitalização, facilita a compreensão e aceitação dos procedimentos, estimula a criança a manter sua capacidade física, diminui a hostilidade do ambiente e proporciona um melhor relacionamento entre a equipe de saúde e a criança.

\section{REFERÊNCIAS}

BRASIL. Conselho Nacional de Saúde. Resolução no 466/12, de 12 de dezembro de 2012. Aprova as diretrizes e normas regulamentadoras das pesquisas envolvendo seres humanos. Diário Oficial da União, Brasília, DF, 2012. Disponível em:< http://conselho.saude.gov.br/resolucoes $>$. Acesso em 02 set. 2017.

CONCEICAO, C.M. et al. Brinquedo terapêutico no preparo da criança para punção venosa ambulatorial: percepção dos pais e acompanhantes. Esc. Anna Nery, Rio de Janeiro, v.15, n.2, p.346-363, 2011. Disponível em: <http://www.scielo.br>. Acesso em: 27 out. 2018.

COFEN. Conselho Federal de Enfermagem. Resolução no 295, de 24 de outubro de 2004. Dispõe sobre a utilização de técnica do brinquedo/brinquedo terapêutico pelo enfermeiro na assistência à criança hospitalizada. Rio de Janeiro, 2004. Disponível em: <http://novo.portalcofen.gov.br>. Acesso em: 27 out. 2018.

CRUZ, D.S.M. et al. Therapeutic toy: integrative review. Journal of Nursing UFPE on line - ISSN: 1981-8963, [S.1.], v.7, n.5, p.1443-1448, 2013. Disponível em: <https://periodicos.ufpe.br/revistas/revistaenfermagem>. Acesso em: 20 out. 2018.

DANSKI, M.T.R. et al. Complicações relacionadas ao uso do cateter venoso periférico: ensaio clínico randomizado. Acta paul. enferm., São Paulo, v.29, n.1, p.84-92, 2016. Disponível em: $<$ http://www.scielo.br>. Acesso em: 26 out. 2018.

FONTES, C.M.B.; MONDINI, C.C.S.D.; MORAES, M.C.A.F.; BACHEGA, M.I.; MAXIMINO, N.P. Utilização do brinquedo terapêutico na assistência à criança hospitalizada. Rev Bras Educ Espec., Rio Grande do Sul, v.16, n.1, p. 95-106, 2010. Disponível em: <http://www.scielo.br>. Acesso em: 04 abril 2019. 
FORTUNATO, J. et al. Escalas de dor no paciente crítico: Uma revisão Integrativa. Revista HUPE, Rio de Janeiro, 2013. Disponível em: <http://www.e-publicacoes.uerj.br>. Acesso em: 03 set. 2107.

KICHE, M.T.; ALMEIDA, F.A. Brinquedo terapêutico: estratégia de alívio da dor e tensão durante o curativo cirúrgico em crianças. Acta paul. enferm., São Paulo, v.22, n.2, p.125-130, 2009. Disponível em: <http://www.scielo.br>. Acesso em: 04 set. 2017.

MAIA, E.B.S.; RIBEIRO, C.A.; BORBA, R.I.H. Compreendendo a sensibilização do enfermeiro para o uso do brinquedo terapêutico na prática assistencial à criança. Rev Esc Enferm USP, São Paulo, v.45, n.4, p.839-846, 2011. Disponível em: <http://www.scielo.br>. Acesso em: 04 abril 2019.

MEDEIROS, G. et al. Brinquedo terapêutico no preparo da criança para punção venosa em pronto socorro. Acta paul. enferm., São Paulo, v.22, n.spe, p.909-915, 2009. Disponível em: $<\mathrm{http}: / /$ www.scielo.br>. Acesso em: 10 ago. 2017.

PONTES, J.E.D. et al. Brinquedo terapêutico: preparando a criança para a vacina. Einstein, São Paulo, São Paulo, v.13, n.2, p.238-242, Junho 2015. Disponível em: <http://www.scielo.br >. Acesso em: 23 out. de 2018.

ROCHA, P.K.; PRADO, M.L.; SILVA, D.M.G.V. Pesquisa convergente assistencial: uso na elaboração de modelos de cuidado de enfermagem. Rev Bras Enferm., v.65, n.6, p.1019-1025, 2012. Disponível em: <http://www.scielo.br >. Acesso em: 04 abril de 2019.

SOUSA, L.C.; DE VITTA, A.; LIMA, J.M.; DE VITTA, F.C.F. The act of playing within the hospital context in the vision of the accompanying persons of the hospitalised children. $J$ Human Growth Develop., v.25, n.1, p.41-49, 2015. Disponível em: <http://www.scielo.br >. Acesso em: 04 abril de 2019.

SOUZA, L.P.S. et al. O Brinquedo terapêutico e o lúdico na visão da equipe de enfermagem. J Health Sci Inst., v.30, n.4, p.354-358, 2012. Disponível em: <http://www.scielo.br >. Acesso em: 04 abril de 2019. 